\title{
El test del Árbol, su aplicación en la exploración de la personalidad en la clínica médico-forense.
}

\author{
Application of the Koch Tree test in the exploration of \\ personality in Forensic Medicine.
}

J.M. Cid Rodriguez ${ }^{1}$ y M. Castellano Arroyo 2

\section{RESUMEN}

En el presente artículo se propone el Test del Árbol como un instrumento muy útil para la exploración médico-forense en casos de violencia familiar, agresiones sexuales y en los casos de víctimas infantiles. Para la interpretación de la prueba se indica la metodología a seguir y la bibliografia necesaria. Como aplicación práctica se expone una pericia sobre una niña víctima de abusos sexuales y los dos adultos denunciados; en el informe se comparan los resultados obtenidos en el Cuestionario Factorial de Personalidad I6-PF de Catell y el análisis del Test del Árbol dibujado por las personas exploradas.

Palabras clave: Abuso infantil. Violencia familiar. Exploración psicológica médico-forense. Test del Árbol de Koch.

\section{ABSTRACT}

In a case of infant sexual abuse a number of psycho-diagnostic tests were performed both in the victim and two men arrested as possible perpetrators. After collecting and analyzing all results, the authors formally propose the "Koch Tree test" as a projective test of forensic utility.

Key words: Infant sexual abuse. Infant sexual abuse. Family violence. Psycho-diagnostic tests in Forensic Medicine. The "Koch Tree Test".

1 Profesor Asociado de la Facultad de Psicología y de la Facultad de Ciencias de la Educación. Universidad de Granada.

2 Catedrática de Medicina Legal y Forense. Universidad de Granada. Médico Forense en situación de excedencia. 


\section{INTRODUCCIÓN:}

La peritación psiquiátrica forense es tarea compleja que exige conocimientos específicos dentro de la especialidad en Medicina Legal y Forense. Actualmente esta prueba pericial puede estar en relación con aspectos muy variados que deben ser tenidos en cuenta. La solicitud puede dirigirse hacia los siguientes extremos:

I. Establecer el grado de imputabilidad de la persona en relación a una conducta de la que es materialmente autora, pero es preciso explorar si es también autora moral de la misma conforme a un adecuado grado de comprensión y a la capacidad de elegir. Generalmente son conductas antijurídicas en las que está previsto que la persona autora responda del daño causado y lo repare.

2. Establecer el grado de capacidad de obrar en relación a disposiciones sobre la propia persona o sobre los bienes. Los temas civiles más comunes al respecto son la validez de contratos, testamentos, decisiones de vivir en un lugar u otro (residencias de ancianos, familiares...), o en determinadas condiciones.

3. En relación con la violencia, ya sea a nivel familiar o fuera de este ámbito, se plantea a menudo como pericia lo siguiente:

a) Estructura de personalidad de la víctima de una agresión. Si se estudia el perfil de personalidad de la víctima, inmediatamente después de la denuncia, servirá para establecer su vulnerabilidad y si existe predisposición o no a afrontar negativamente el hecho, de manera que supere el acontecimiento a través de una "victimización", nefasta para ella [I].

b) Estructura de la personalidad en personas agresoras o autoras de una conducta hostil y psíquicamente agresiva. A través de estos datos se podrá conocer lo que en esa persona es caracterial, temperamental o cultural, y se podrán modificar actitudes y conductas de cara al futuro.

c) Establecer las secuelas de tipo psíquico que padece una persona sometida a un maltrato psíquico y/o físico durante un tiempo más o menos prolongado.

d) Exploración integral de una persona lesionada (accidente de tráfico, de trabajo, etc.) en la que las lesiones físicas y las secuelas que hayan podido originar, se acompañan de síntomas psíquicos que deben valorarse en dos direcciones: I) iSon consecuencia de las lesiones físicas?. 2) iEran rasgos preexistentes y por tanto han podido influir, incluso en la evolución de las lesiones físicas?.

Sin embargo, aunque las pericias parecen muy diversas, desde el punto de vista médicoforense siempre se tratará del mismo acto médico, la exploración de una persona en la que hay que establecer uno o varios de estos extremos:

- Nivel de inteligencia o capacidad comprensiva y de juicio.

- Integridad de la voluntad, tanto para los aspectos de elección libre como para la capacidad de controlar impulsos no lícitos.

- Rasgos importantes de su personalidad relacionados con: la afectividad, la estabilidad emocional o fuerza del yo, su nivel de introversión o extroversión, su seriedad o su entusiasmo, su impulsividad, la conflictividad con su propia imagen, su nivel de autoestima, su franqueza o suspicacia, su tendencia a experimentar ansiedad y su grado de tensión emocional, la calidad triste o alegre de su estado de ánimo, la forma en que superó su pasado y sus expectativas con respecto al futuro, etc. 
Para todas estas cuestiones encuentra la pericia médico-psicológica-psiquiátrica respuesta en:

I) Una exploración completa que englobe una entrevista personal de la que se extraiga la psicobiografía de la persona.

2) Exploración psicodiagnóstica a través de las correspondientes baterías de tests mentales con tests de inteligencia, de personalidad, proyectivos, escalas de evaluación de conducta, etc.

Lo dicho hasta aquí nos lleva a concluir que la exploración médico-forense plantea unas exigencias que pueden hacer de ella una pericia compleja, experta y llena de responsabilidad.

En relación con esto, el presente artículo tiene dos objetivos:

I. Proponer el test del Árbol como un instrumento inicial en la exploración médico forense, especialmente útil en determinados casos como la violencia familiar, los delitos contra la libertad sexual, exploraciones infantiles, etc. El hecho de que estas personas puedan someterse a la exploración con recelo o temor hace que el test del árbol sea un instrumento que facilita el contacto inicial en la entrevista y el posterior abordaje del caso.

2. El segundo objetivo es plantear el análisis de un caso que sirva como ejemplo a lo que acabamos de decir. Se trata de una denuncia por abusos sexuales en los que la víctima es una niña de 12 años (O.G.C.) y los acusados son dos vecinos del pueblo: M.S.M. de 60 años y J.D.O. de 78 años. El estudio de las tres personas implicadas se hizo a tarvés de entrevistas personales y de una batería de tests y escalas bastante completa. Sin embargo, en el presente artículo presentamos solo el Test del Árbol realizado por cada una de las tres personas y el resultado del Cuestionario de Personalidad I 6-PF de Catell, al objeto de comparar la información obtenida en un Cuestionario sistematizado y validado frente a la información que ofrece el Test del Árbol.

El Test del Árbol fue propuesto por K. Koch en 1957 [2, 3] y posteriormente varios autores contribuyeron a su sistematización y aplicación en diferentes grupos de pacientes, fue el caso de R. Stora [4], M. Passi [5], F. Abad [6], M. Xandro [7] y J.Ma Cid [8].

El Test consiste en indicar al probando, al mismo tiempo que se le ofrece un folio en blanco y un lápiz, que dibuje un árbol frutal. Ante una propuesta tan poco directiva el resultado es totalmente personal y muy variado.

Su fundamento está en la proyección inconsciente de la propia persona en el dibujo en el que se reflejan los tres planos dinámicos: El inconsciente (raíces y suelo), el preconsciente (tronco) y el consciente (copa), lo que también puede hacerse coincidir con el ego, el yo y el superyo. La forma en que se dibuja el suelo o base, horizonte, ramas, frutos, follaje, etc. son de la mayor importancia interpretativa.

Esta es una prueba que tanto en el caso de las víctimas como de los agresores puede dar datos especialemnte significativos que integrados con los hechos, la entrevista y otros instrumentos de exploración completen una visión holística del caso. Nosotros ya hicimos una primera aplicación a mujeres víctimas de violencia familiar [9], estudio que continuamos en la actualidad.

En el presente artículo queremos proponer la aplicación del Test junto a la forma sistemática de recogida e interpretación de las variables, siguiendo las propuestas de los autores antes citados, al objeto de que se convierta en un instrumento útil en la exploración médico-forense. 


\section{MATERIAL Y MÉTODO:}

I. El Cuestionario de Personalidad I6-PF de Catell [I0].

2. El material necesario, como ya se ha mencionado, se reduce a un folio en blanco y un lápiz que se le ofrece al probando con la siguiente consigna: "Dibuje un árbol frutal". En el caso de que el perito pueda observar a la persona mientras dibuja podrá anotar la secuencia del dibujo, su actitud ante la propuesta y la propia realización, su fuerza al dibujar, etc

Posteriormente, el análisis del dibujo se hará teniendo en cuenta la "Ficha Técnica" de recogida de datos que se propone en el presente artículo, y para la interpretación debe seguirse el libro ya citado titulado "El Test del Árbol. Evaluación Psicológica (Índice de Variables de Interpretación). José Ma Cid Rodriguez, 1998. En este libro el autor ha reunido todas las aportaciones que los investigadores del Test del Árbol, citados anteriormente, han realizado. La recogida sistemática de datos y su interpretación se atiene al siguiente esquema:

A) Formas Primarias o Rudimentarias. Estas las describió Koch en número de 13 y en el libro están recogidas en el "Índice de las Formas Rudimentarias". A continuación, se expone el "Esquema de las Formas Rudimentarias" en el que Koch reproduce mediante dibujos las 13 formas mencionadas; en estos dibujos se reproduce tanto el esquema global del árbol como el aspecto específico de la base del tronco o las raíces. Y en tercer lugar el libro recoge los "Datos Estadísticos y de Interpretación" de todas ellas tal y como fueron propuestas y defendidas por el autor. De los autores mencionados Koch fue el único en describir, valorar e interpretar las "Formas Primarias y Rudimentarias".

La segunda parte del estudio de las "Características Formales" la componen:

B) Elementos Esenciales Morfológicos. Son 7 variables imprescindibles para que el dibujo se identifique con un árbol, aunque pueden estar o no todas ellas presentes en un determinado dibujo: Estos son: suelo, raíces, tronco, ramas, copa, follaje y accesorios.

En esta parte, el libro contiene una serie sucesiva de Tablas ("Índices") uno por cada "Elemento Esencial Morfológico", en el que se recogen las posibles variaciones de cada elemento (Items), según el criterio de cada uno de los autores estudiosos del Test del Árbol. Cada autor le asigna un número de orden en su publicación original a los items (a excepción de Kock cuyos items los hemos identificado con el número de la página de su libro en la que aparece).

A continuación, en el libro se muestran sucesivos dibujos junto a los cuales aparece la valoración interpretativa que los autores citados han realizado.

A la presentación del significado de las variables en particular, le siguen los items ordenados en la forma que propusieron cronológicamente los autores según las fechas de sus publicaciones.

La mayor parte de los items se acompañan del dibujo/s en los que se hace la interpretación propuesta por cada autor empleando la primera letra del nombre del mismo: K:Koch, P: Passi, A:Abad y X:Xandró. La amplitud de la explicación varía de un autor a otro, pues mientras los hay que agrupan los items en un número limitado de factores o de rasgos básicos, otros, en cambio se extienden en sus conclusiones, si bien esta particularidad es más destacada en $\mathrm{KOCH}$ por ser el inventor del Test.

Seguidamente se valoran los Conflictos Expresivos que pueden ser:

A) Proyectivos: Movimiento, Tipo de Línea, Trazos Dominantes, Posición o situación, Tamaño o dimensión, Alturas y Anchuras.

B) Estéticos: Sombreados, Formas Globales, Estilo, e Impresión General. 
Son los que los autores aportan de forma conjunta con el resto de los Elementos Morfológicos del Test en sus respectivos textos, y que en el libro se ha preferido distribuirlos en función de sus características, así como por las relaciones que se destacan en el desarrollo proyectivo - psicomotor, y de las coordenadas espacio - temporales, y estéticas, que se proyectan y expresan en el momento en el que se efectúa la propia ejecución de la prueba.

En la medida en que el perito tenga un mejor conocimiento del Test, así como de las bases psicológico-psiquiátricas en las que se apoya, podrá obtener del mismo mayor rendimiento, complementando cada uno de los escalones siguientes:

I. Índice de las Variables de Interpretación (I.V.I.). A partir de los índices, se anotan cada una de las variables e items que estén presentes en el dibujo. De forma secuencial debe consignarse el número de la variable y de los items asignados en el libro, acompañado del epígrafe que le corresponde.

2. Índice de los Contenidos de las Variables de Interpretación (I.C.V.I.). En este apartado, se deben detallar los contenidos psicológicos e interpretativos con los que los investigadores del Test del Árbol han designado a sus variables e items; estos se clasifican en: Acción (A), Sentimiento (S), y Pensamiento $(P)$, atendiendo a las definiciones aportadas por las Tópicos de la Estructura Psicodinámica de la Personalidad, dirigiéndolo hacia la Hipótesis Convergente.

3. Evaluación Psicológica e Interpretación (E.P.I.). Aquí se tienen en cuenta las partes estructurales del árbol: los elementos morfológicos que según los estudiosos del test constituyen la estructura psicodinámica formulada en la Hipótesis Convergente, y acompañados por la interpretación de los Conflictos Expresivos observados en el dibujo. Todo ello conduce a establecer el perfil psicológico de la personalidad.

4. Valoración Personal de la Experiencia (V.P.E.). Este último apartado puede aplicarse en la relación terapeútica con el probando. En casos de víctimas de agresiones, violencia crónica, etc., en el proceso de afrontamiento es muy útil ayudarle a enfrentarse con la realidad desde su propia manera de ser y de vivir el hecho. Por ello, explicarle algunos extremos de su dibujo facilita la comunicación y apoya la terapia de esclarecimiento que puede proporcionarse como complemento al mero informe.

\section{RESULTADOS:}

La aplicación práctica del Test del Árbol la vemos a través de un Informe Médico-forense solicitado por la Administración de Justicia en un caso real y realizado sobre tres personas: I. Olga G. C. De 12 años; 2. Manuel S.M.. de 60 años; y 3. Jesús D.O. de 78 años.

\section{OLGA G. C. NIÑA DE 12 AÑOS.}

Olga vive en un pueblo de 492 habitantes y su madre ha denunciado a dos vecinos indicando que esta ha sido víctima de tocamientos en pecho y genitales con la petición de que participe en actos masturbatorios. El ambiente familiar de la niña puede resumirse en los siguientes datos: vive con sus padres ambos alcohólicos y su madre padece un grado leve de retraso mental. Va al colegio, pero hace una vida poco controlada, sin disciplina ni normas de socialización. La sexualidad la ha vivido desde su infancia como algo natural ya que su madre le refería las relaciones sexuales e incluso presenciaba las mantenidas por sus padres. Eso ha hecho que para Olga el contacto sexual sea como una forma más de relación entre las personas, por lo que su comportamiento ha sido colaborador y hasta incitador, en ocasiones hacia los hombres acusados u otros del pueblo, que al 
parecer rechazaron la relación. No tiene conciencia de víctima y respecto a sus agresores dice: "...yo paso de ellos, si me los cruzo les vuelvo la cara". Cuando hablamos con la niña se presenta en el despacho del Centro educativo donde sigue su escolarización en una actitud hostil (gesto mohino, mira de reojo, cara vuelta) y cuando se sienta le decimos: "Me han dicho que dibujas muy bien iQuieres pintarme un árbol frutal?'. La niña cogió el folio blanco y dibujó el árbol no I. El estudio del mismo lo hacemos más adelante. Tras esto, y a medida que trascurre la conversación se muestra más confiada, por lo que a lo largo de la conversación le solicitamos que dibuje lo que ella quiera a lo que responde con el dibujo $\mathrm{n}^{\circ} 2$, que consiste en un paisaje en el que el dibujo central es una casa, con un árbol próximo al margen izquierdo y un cielo con sol radiante y nubes que llegan hasta el margen derecho. Este árbol es el que analizamos como $n^{\circ} 2$ y comparamos con el $n^{\circ}$ I en la discusión.

ANÁLISIS DE LOS DIBUJOS DE Olga G.C. El análisis se recoge en la "FICHA DE RECOGIDA DE DATOS" que proponemos en el presente artículo para el test del Árbol, en el mismo se reproduce cada dibujo en la misma escala que tiene el folio y el dibujo realizado.

\section{NIÑA VÍCTIMA DE ABUSOS SEXUALES}

\section{Datos Personales:}

Nombre: Olga G.C. Edad: 12 años. Arbol Dibujado: Manzano. Años: 2.

Situación: Víctima de abuso sexual.

\section{Dibujo $\mathrm{n}^{\circ}$ I: II. Índice de las Variables de} Interpretación:

a) Formas Primarias: 7, 8, 12.

b) Elementos Morfológicos:

I. Suelo: $4,5,6$.

2. Raíces:

3. Tronco: $2,8,21,24,29,43,53$, $59,60,61,64$.

4. Ramas: 47

5. Copa: I, 5, I0,27.

6. Follaje: $|1|, \mid 2,19,24,30,31,37,42,51,52$.

7. Accesorios: 8,10,11.

\section{III. Índice de Conflictos Expresivos:}

a) Proyectivos:

I. Movimiento:

2. Tipo de Línea: $2,14$.

3. Trazos Dominantes: 13, 19,31,44.

4. Posición: 2, 10,13.

5. Tamaño: 3.

6. Alturas y Anchura: 3,5,I.

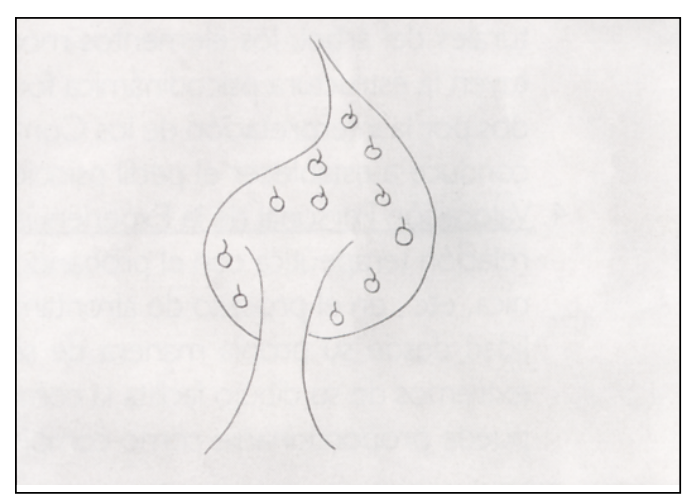

b) Estéticos:

7. Sombreados:

8. Formas Globales: I , 2,3

9. Estilo: 5. 1. 2.

I0. Impresión General:

Claro,flotante,simple,

Expresivo, peculiar.

\section{Perfíl de la Personalidad: Evaluación Psicológica:}

Inconsciente: Las formas primarias: desplazamientos espaciales, copa amalgamada, con frutos grandes estereotipados, manifiestan cierta debilidad mental o retraso en el aprendizaje. 
Preconsciente: Las líneas ascendentes y descendentes del tronco reflejan reserva y retraimiento, aunque su forma ascendente es indicativa de espíritu emprendedor. Su grosor significa actividad. La base abierta y sin suelo indica el conflicto impulsivo en el que vive. El estar hecho de una pieza refleja infantilismo. La inclinación a la derecha señala búsqueda de apoyo, lo cual se completa con la falta de base que interpretamos en el mismo sentido; igual que el ensanchamiento en la base. El estrechamiento central reitera su expresión inhibida, aunque al estar abierto y unido a la copa expresa cierta capacidad de concentración y una inteligencia adecuada. La inquietud sexual y la falta de contacto se observan en que el tronco entra en la copa y está suspendido en el suelo. La falta de ramas reitera la dificultad de trabar relaciones.

Consciente: El tamaño excesivo de la copa revela egocentrismo y su aspecto globuloso falta de apoyo. Las bolsas en ambos lados denotan su situación actual, falta de energía y voluntad. En principio, el penacho dirigido hacia arriba podría significar fantasía y hasta tendencia a la fabulación; sin embargo, el contexto en el que dibuja ese penacho, que se comentará en la discusión, es indicativo de agresividad hacia la persona que la explora y, desde luego, tendencia a la fantasía y un mundo aislado y algo autista en los afectos verdaderos. La permanencia en guardia se exhibe en las curvas y su retorno al pasado en su orientación a la izquierda del final de la copa. Es sensible al medio y desea compensar un sentimiento de insuficiencia, por estar abierta la copa y se desborda en lo alto. Los frutos estereotipados, aislados denotan inhibición e infantilismo.

Conflictos: Las curvas denotan la sensibilidad, y las líneas continuas y sueltas la dificultad de contacto. La fuerte presión y su rapidez detectan el conflicto vivido, y su trazo fino, la abulia y la falta de fuerza. Se repite su deseo de protección al colocar el dibujo a la izquierda con tendencia al centro, pero la inversión de la hoja enseña su espíritu independiente, su tamaño denota la falta de una exacta valoración de sí misma, y sus alturas y anchura, dicha búsqueda de adaptación y la duda de su inteligencia que se remarca con los vacíos en el interior de la copa. La forma es la de un árbol impropio, que en nuestra opinión surge motivado por la dirección agresiva hacia la persona que la explora sentada enfrente. El alejamiento de la realidad se expone en su estilo extraño e infantil, con falta de contacto por el espacio vacío de la izquierda (la madre) y por su forma desproporcionada.

\section{DIBUJO No 2 DE Olga G.B.}

Como se ha dicho, este dibujo lo realizó la niña tras más de una hora de entrevista, cuando ya se había diluido su resistencia y temor y ante la propuesta "Ahora me vas a dibujar lo que tu quieras". El dibujo contiene una casa como elemento central, un árbol a la izquierda que representa a ella misma y un espacio superior que representan sus fantasías y expectativos.

Del mismo analizamos fundamentalmente el árbol, para compararlo con el primero, y dedicamos unas palabras al resto por su interés en la situación médico-psicológica de la niña.

\section{NIÑA VÍCTIMA DE ABUSOS SEXUALES}

\section{Datos Personales:}

Nombre: Olga G.C. Edad: 12 años.

Dibujo con una casa, un árbol a la izquierda y cielo con nubes y sol. Se analiza el árbol.

Situación: Víctima de abuso sexual. 


\section{Dibujo $\mathrm{n}^{\circ}$ 2. II. Indice de las Variables de Interpretación:}

a) Formas Primarias: 7,8, 12.

b) Elementos Morfológicos:

I. Suelo: 4,5,7, 17,

2. Raíces:

3. Tronco: igual que el primer dibujo (no 61, si 62).

4. Ramas: igual

5. Copa: $1,5,10,12,18,20$.

6. Follaje: 7,8, II, 12, 17, 19,30,40.

7. Accesorios: 3,8,10,11.

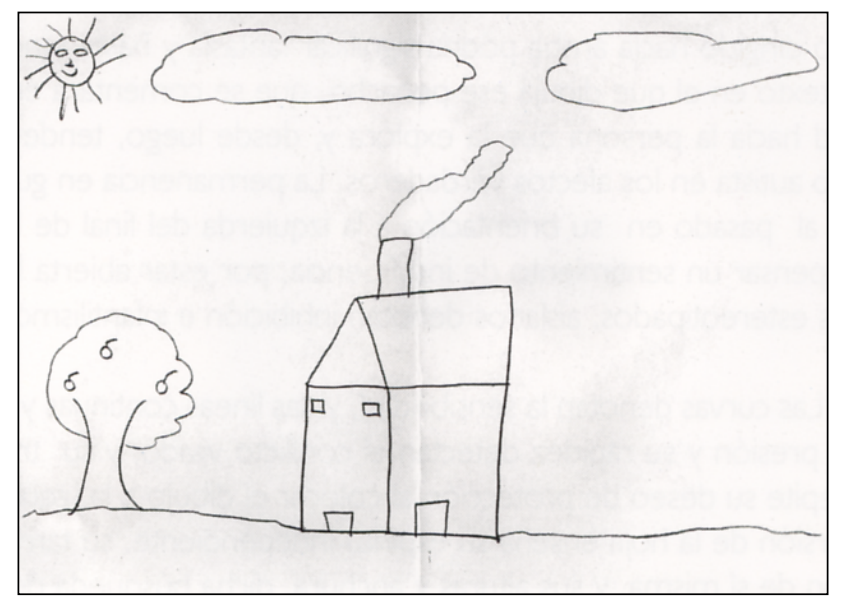

\section{III. Índice de Conflictos Expresivos:}

a) Proyectivos:

I. Movimiento:

2. Tipo de Línea: $2,6,12,14$.

3. Trazos Dominantes: $1,2,19,31,32,47$.

4. Posición: 1,9,13.

5. Tamaño: 4.

6. Alturas y Anchura: 1.2.1. b) Estéticos:

7. Sombreados: 10

8. Formas Globales: 2,3.

9. Estilo: I.I.2.

I0. Impresión General:

Claro, tierno,alado, expresivo,

dinámico.

\section{Perfíl de la Personalidad. Evaluación Psicológica:}

Inconsciente: Aquí aparece un suelo ascendente hacia la izquierda que nos revela el sentimiento de soledad, y su leve acentuación en zig-zag su afán de realización, soledad que se reafirma al estar el tronco separado del suelo por un único trazo.

Preconsciente: Muy similar al que realizó en el primer dibujo ( tronco sin ramas).

Consciente: La copa varía al estar formada por arcadas que reflejan la obsequiosidad de la niña, al ser doble y ágil, presenta la comprensión de los problemas. El follaje es filiforme que es una manera de eludir los problemas y en curvas abiertas y en arcadas en las que se expresa su recepti- 
vidad, pero también su desconfianza. Se orienta hacia la derecha en donde sitúa la casa en la que busca apoyo, al estar cerrado protege su intimidad y sus pertenencias. El árbol acompaña a una casa más grande que analizaremos más adelante.

Conflictos: El trazo es más fino en la copa del árbol, en el suelo, las nubes y el humo, que en el resto de los elementos que lo acompañan; los trazos de la casa, del tronco y del sol son más fuertes. El árbol está situado junto al margen izquierdo del papel, lo que revela una actitud vinculada al pasado y aferrada a la madre, al estar situado en un plano bajo denota la sensación de abandono y soledad. El tamaño aquí es más pequeño que en el dibujo $n^{\circ} I$, y sus alturas y anchura ponen de manifiesto una actitud a la defensiva. Los vacíos de la copa se repiten en este dibujo y la irregularidad, así como la imagen antropomorfa (cara), en cuyas interpretaciones coincidimos con el dibujo anterior. El estilo es escueto e infantil.

El hecho de que invierta el folio y lo utilice en sentido horizontal indica cierto nivel de independencia y oposición a la norma.

Brevemente dedicamos unas líneas a la interpretación del resto del dibujo, aunque en el Informe médico-forense original, se hacía una interpretación más completa del mismo, junto a otro de la figura humana y un cuarto de tema libre que no hemos incluido en el artículo al tratar este sobre la interpretación del Test del Árbol.

Se trata de una casa apacible, con chimenea y humo (calor de hogar) y tejado grande y protector, significa para ella la seguridad de su casa (lo cual verbaliza en la entrevista ("quiero volver a mi casa con mis padres"). Sin embargo, es una casa con puertas y ventanas pequeñas cerradas y sin camino de acceso. Está cerrada y no invita a penetrar en ella. Es su propio egocentrismo, aislamiento, reserva e introversión. También la casa tiene una imagen antropomorfa (cara que mira hacia el árbol) en correspondencia con el árbol también antropomorfo. El conjunto representa su círculo familiar ella y su hogar mirándose mutuamente. El árbol está a la izquierda, vinculado al pasado, mira hacia el hogar y la madre y la imagen paterna queda representada por el sol, que también está a la izquierda, aunque arriba, sonriente e iluminando su infancia y su pasado de los que ella no quiere soltarse.

Todo el plano central del dibujo, en el sentido horizontal, que como hemos dicho representa la realidad actual, se corta con una línea vertical frente al futuro, al que se cierra y teme.

Sin embargo el plano de sus fantasías (superior) está ocupado por las nubes que sí llegan hasta la derecha, indicando que, a nivel fantástico, si mantiene expectativas de futuro y de salir de su realidad actual.

\section{Manuel S.M. VARón de 60 años.}

Está denunciado por abusos sexuales contra Olga G.C. Es un hombre casado y tiene tres hijos y dos nietos. Es bebedor excesivo regular y refiere al hablar de sus hábitos que bebe unos 2 litros de vino tinto al día y alguna copa de aguardiente. Manuel aparece como un hombre torpe, contesta con pocas palabras y se muestra retraído y a la defensiva. Trabaja en el campo como guarda de un coto $y$, en general mantiene un gran aislamiento social. Por su actitud no parece darle mucha importancia a los hechos de que está denunciado y niega que haya tocado a la niña, pero al mismo tiempo se queja de que la niña no tenía control de su familia y que se metía en su casa preguntando por sus nietos. 
De su exploración psicodiagnóstica reproducimos los resultados del Cuestionario Factorial de Personalidad I6-PF de Catell.

\section{Cuestionario de Personalidad 16-PF de Catell}

- Factor A (Afectividad). Puntuación: 2. Persona que tiende a ser dura, fría, y alejada.

- Factor C (Estabilidad emocional, fuerza del Yo). Puntuación: 4. Tolera mal las contrariedades, poca estabilidad emocional, poca fuerza del yo.

- Factor E (Sumisión/Dominancia). Puntuación: 8. Dominante, autoritario con los demás y agresivo.

- Factor F (Sobriedad/Entusiasmo). Puntuación: 4. Más bien serio, taciturno y poco expresivo.

- Factor G (Poca/Mucha fuerza del Superego). Puntuación: 2. Despreocupado de las forma y normas sociales, acepta pocas obligaciones, bajo nivel de conciencia moral.

- Factor H (Timidez/Atrevimiento). Puntuación: I. Persona alejada, retraída, reprimida, al margen de la actividad social.

- Factor I (Dureza/Sensibilidad). Puntuación: I0. Persona impresionable, labilidad afectiva, excesivamente afectable.

- Factor L (Confianza/Suspicacia). Puntuación: 9. Suspicaz, desconfiado, no se integra bien en el grupo, despegado.

- Factor M (Practicidad/Imaginación). Puntuación: 7. Poco práctico, despreocupado de la realidad.

- Factor N (Franqueza/Astucia). Puntuación: 7. Tendencia a la astucia, poca franqueza.

- Factor O (Adecuación imperturbable/Tendencia a la culpabilidad). Puntuación: 7. Ante las dificultades tendencia infantil a la ansiedad.

- Factor QI (Conservadurismo/Radicalismo). Puntuación: 6. Equilibrio entre el ser conservador o radical.

- Factor Q2 (Dependencia/Autosuficiencia). Puntuación: 8. Temperamentalmente independiente, actúa por su cuenta.

- Factor Q3 (Autoconflictivo/Buena Autoimagen). Puntuación: 2. Autoconflictivo, orientada por sus propias necesidades, afectivamente desajustado

- Factor Q4 (Relajación/Tensión emocional). Puntuación 4. Bajo nivel de tensión y "ansiedad flotante".

Como resumen de su perfil de personalidad, evaluada por el I6-PF de Catell, podemos decir que es un hombre frío y poco afectivo [2]. Retraído, al margen de la sociedad [I]. Inestable emocionalmente y lábil afectivamente [4] y [10], en este aspecto puede influir el deterioro que sin duda le ha producido su alcoholismo. Dominante y agresivo [8]. Poco respetuoso con las normas sociales [2]. Suspicaz [9]. Poca franqueza y tendencia a la mentira [7]. En conflicto consigo mismo [2]. Bajo nivel de ansiedad (seguramente esta está mitigada por su consumo de alcohol y su baja autocrítica). 


\section{TEST DEL ÁRBOL:}

HOMBRE: PRESUNTO AUTOR DE ABUSOS SEXUALES A UNA MENOR

\section{Datos Personales:}

Nombre:Manuel S.M. Edad: 60 años

Árbol dibujado: olivo. Años: $14-19$

Situación: Acusado de abuso sexual a menor.

\section{II. Índice de las Variables de Interpretación:}

a) Formas Primarias: 1, 2, 6, 7, 10, 12, 13.

b) Elementos Morfológicos:

I. Suelo: 8.

2. Raíces:

3. Tronco: $1,21,24,48,50,60,61$.

4. Ramas: 6, II, |4,24,27.

5. Copa: 14,23 .

6. Follaje: $4,11,15,30,42,44$.

7. Accesorios: $8,10$.

\section{III. Índice de Conflictos Expresivos:}

a) Proyectivos:

I. Movimiento:

2.Tipo de Línea: $1,3,7,14$.

3. Trazos Dominantes: 1,5,29.

4. Posición: 2,8, 14 .

5. Tamaño: 4.

6. Alturas y Anchura: I. I. I.

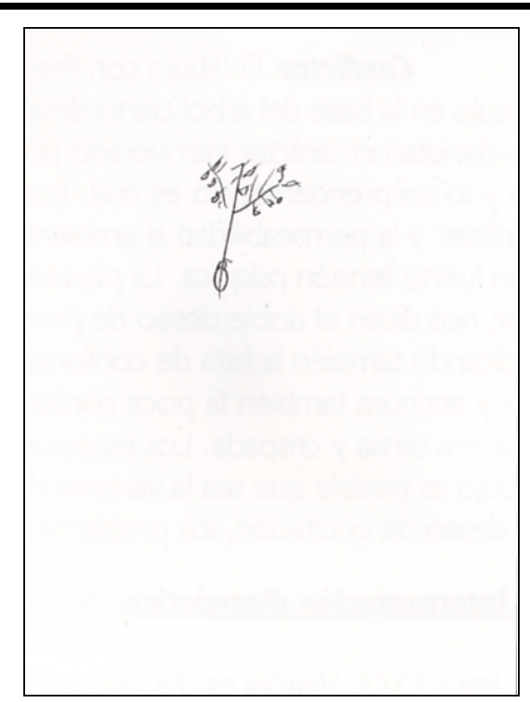

b) Estéticos:

7. Sombreados: 10

8. Formas Globales:2.

9. Estilo: 2.2.2.

10. Impresión General: frío, simple,

Pobre, recio, seco

\section{Perfíl de la Personalidad. Evaluación Psicológica:}

Inconsciente: Las formas primarias: tronco en raya sin copa con ramas cortas, ramas en raya en sentido ascendente, desplazamientos espaciales formas agregada y degenerada, muestran lo rudimentario auténtico del dibujo propio de adultos con estado regresivo, debilidad mental o deterioro, signos de atraso en la evolución intelectual y afectiva. El suelo circunscrito muestra el estado de aislamiento pero, también de egocentrismo con posibles mentiras con ocultación o cobardía.

Preconsciente: El tronco en raya simple, de un solo trazo, muestra la introversión y la inhibición, y al estar dibujado de una pieza demuestra el infantilismo; inclinado a la derecha, con simetría angular aguda con las ramas, denota el probable problema moral que sufre el sujeto, con inquietud en cuanto a la sexualidad y la falta de contacto con el mundo.

Consciente: Las ramas delgadas de un solo trazo, dirigidas hacia arriba con interrupciones y rectas, nos revelan el conflicto social, la excitabilidad, así como la falta de madurez intelectual o afectiva, que al estar interrumpidas con el tronco, demuestran la veleidad del sujeto y las manías ocasionales que sufre con tendencia exhibicionista y ambivalente. Las ramas con forma de varas orientadas del centro, hacia fuera representan la agresividad y la búsqueda de contacto con la realidad y la falta de perseverancia. El roto u omisión es que esconde algo. El follaje con redondeles remarca 
el carácter oral y la tendencia a una organización más o menos sistemática, y los medios rombos de los trazos de las ramas con el tronco, el deseo de parecer seguro de sí mismo, y su orientación hacia la derecha el deseo de un apoyo firme, de deseos insatisfechos que el sujeto niega. Los frutos estereotipados demuestran el carácter inmaduro y coqueto de la persona.

Conflictos: El dibujo con líneas rectas y con ángulo activo, revela la fuerza y el egoísmo, por el círculo en la base del árbol cierto distanciamiento con los demás, tal vez retraso mental. Las líneas sueltas denotan el carácter introvertido poco persistente y desconfiado, con cierta búsqueda de lo novedoso y lo sorprendente, no es real. Los trazos de fuerte presión muy rectos, reiteran dicha dureza de carácter y la permeabilidad al ambiente por la porosidad que presenta en el lado izquierdo del tronco con fuerte tensión psíquica. La posición a la izquierda con tendencia al centro en la parte y mitad superior, nos dicen el doble deseo de protección y de dependencia y su deseo de imponerse a los demás, indicando también la falta de confianza. Su tamaño pequeño refleja la inhibición de impulsos, y las alturas y anchura también la poca confianza en sí mismo en reflexionar permaneciendo a la defensiva de manera tensa y crispada. Los espacios vacíos indican carencias en algún sentido, y la forma regular del dibujo es posible que sea la variante de un síntoma; su estilo incompleto, torpe y deforme, nos resaltan el deseo de ocultación, los problemas de relación y cierto desequilibrio de la personalidad.

V. Interpretación diagnóstica: Bebedor excesivo regular. Ausencia de trastornos mentales.

\section{JESÚS D.O. VARÓN DE 78 AÑOS.}

Casado con una hija y tres nietos. Aparece preocupado y afectado por la denuncia. Niega que haya tocado a la niña, aunque sí reconoce habérsela encontrado en ocasiones en los alrededores del pueblo. Se muestra triste, inhibido y describe con detalle las enfermedades y dolencias que tiene, como en un intento de "dar lástima" por la situación de denuncia en la que está, y que le está afectando mucho familiar y socialmente. Al preguntarle por sus actividades nos refiere que a raíz de los hechos casi no sale de casa, no va al club del jubilado, ni contacta con los vecinos, porque se siente avergonzado ante los comentarios.

\section{Cuestionario de Personalidad I6-PF de Catell}

- Factor A (Afectividad). Puntuación: 3. Persona alejada y baja afectividad.

- Factor C (Estabilidad emocional, fuerza del Yo). Puntuación: 2. Emocionalmente inestable, se afecta fácilmente

- Factor E (Sumisión/Dominancia). Puntuación: 6. Equilibrio entre sumisión y dominancia.

- Factor F (Sobriedad/Entusiasmo). Puntuación: 4. Persona seria, algo reprimida y reticente.

- Fáctor G (Poca/Mucha fuerza del Superego). Puntuación: 4. Poco preocupado por las formas sociales.

- Factor H (Timidez/Atrevimiento). Puntuación: 2. Tímido e introvertido, alejado y cauteloso.

- Factor I (Dureza/Sensibilidad). Puntuación: 9. Muy sensible, turbable y afectable por los sentimientos.

- Factor L (Confianza/Suspicacia). Puntuación: 6. Equilibrio entre la confianza y la suspicacia.

- Factor M (Practicidad/Imaginación). Puntuación: 4. Persona práctica, muy primitiva y elemental con poca imaginación y fantasía.

- Factor N (Franqueza/Astucia). Puntuación: 6. Equilibrio entre la naturalidad sinceridad y la astucia.

- Factor O (Adecuación imperturbable/Tendencia a la culpabilidad). Puntuación: 6. Equilibrio entre ser tranquilo y aprensivo. 
- Factor QI (Conservadurismo/Radicalismo). Puntuación: 6. Equilibrio entre el respeto a lo tradicional y el gusto por lo nuevo.

- Factor Q2 (Dependencia/Autosuficiencia). Puntuación: 6. Equilibrio entre la cohesión al grupo y la independencia-autosuficiencia.

- Factor Q3 (Autoconflictivo/Buena Autoimagen). Puntuación: 5. Aceptable autopercepción de sí mismo y de su propia imagen y autoestima.

- Factor Q4 (Relajación/Tensión). Puntuación: 4. Más bien tranquilo y relajado con bajo nivel de ansiedad

Como resumen de la personalidad o manera de ser de Jesús D.O., podemos decir que es un hombre de 78 años, con un nivel de inteligencia bajo y unos intereses limitados al huerto y sus animales. Es tímido y reservado [2], inestable emocionalmente [3] y muy sensible y afectable por los sentimientos [9]. Es posible que su inestabilidad emocional y sensibilidad hayan aumentando en los últimos años, debido a la edad, ya que la vejez produce a menudo una mayor sensibilidad, y labilidad-inestabilidad afectiva. Su nivel de ansiedad-excitación es bajo [4].

\section{TEST DEL ÁRBOL}

\section{HOMBRE DE 78 AÑOS DENUNCIADO POR ABUSOS SEXUALES A UNA MENOR}

\section{Datos Personales:}

Nombre: Jesús D.O. Edad: 78 años

Árbol dibujado: ciruelo. Años: 20

Situación: Abuso sexual a menor.

\section{II. Índice de las Variables de Interpretación:}

a) Formas Primarias: 7,8, 13.

b) Elementos Morfológicos:

I. Suelo: 15.

2. Raíces: 1,4 .

3. Tronco: $3,6,8,21,24,36,59,61$.

4. Ramas: 47.

5. Copa: $5, \mid 8,21,23$.

6. Follaje: $2,4,5,6,11,13,33,41,42,43$.

7. Accesorios: $9,10$.

\section{III. Índice de Conflictos Expresivos:}

a) Proyectivos:

I. Movimiento:

2. Tipo de Línea: I,2,14.

3. Trazos Dominantes: 5.

4. Posición: $1,8,14$.

5. Tamaño: 4.

6. Alturas y Anchura: I.I.I.

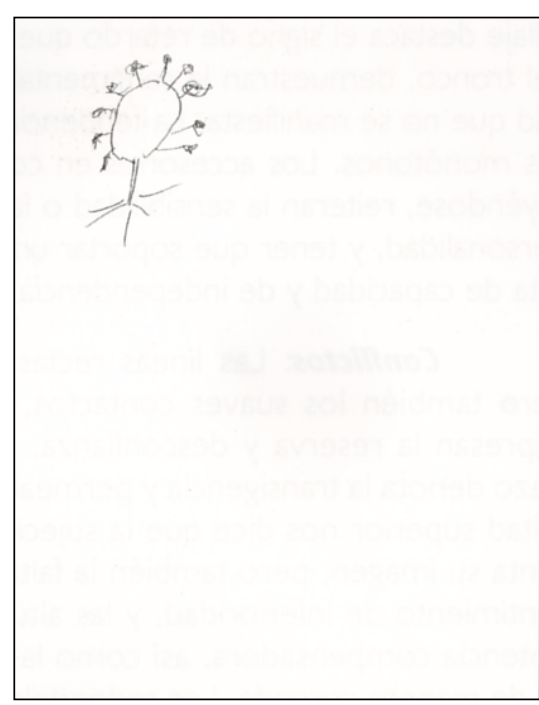

b) Estéticos:

7. Sombreados: 9, 10, 20

8. Formas Globales: 1,2,8.

9. Estilo: 2.2.2.

10.Impresión General: confuso,

Tosco, extraño, pobre, frío. 


\section{Perfíl de la Personalidad. Evaluación Psicológica:}

Inconciente: Las formas primarias del árbol como rueda solar, con desplazamientos espaciales y degenerada, indican la debilidad mental y regresiva que el sujeto padece. El suelo con trazos diversos refleja que se impone una norma personal, y las raíces de un solo trazo y de igual altura que el tronco, denotan el comportamiento infantil y el deseo de conocer lo que se mantiene en secreto con una curiosidad mayor que constituye un problema.

Preconsciente: El tronco que se estrecha en la base da la impresión de inseguridad en un medio que no le proporciona el apoyo deseado, y su delgadez la pasividad en su comportamiento, si bien de base abierta comporta la impulsividad y emotividad que destacan el conflicto social que sufre; al estar hecho de una sola pieza necesita compensar la inseguridad interior y la inclinación a la derecha, su ardor y agresividad, al ser bajo y corto también expresa su sencillez y la falta de contacto con el mundo que se repite en la ausencia de ramas que destacan su actitud de rechazo o de defensa ante la dificultad para establecer relaciones.

Consciente: La copa exagerada de tamaño, muestra su difícil control de la fantasía y el exhibicionismo, su forma esférica manifiesta su tendencia a lo fantástico y a la falta de sentido constructivo, viviendo con ilusiones, mostrando un carácter pueril con miedo a la vida real, su trazo torpe e incoherente denota la inmadurez de la personalidad, y el roto $u$ omisión en la derecha de la copa, el que esconda algo con probables sentimientos de inferioridad. El árbol es irreal, y manifiesta la confusión de ideas y huida de la realidad, y los redondeles demuestra su oralidad, sintiéndose por el péndulo izquierdo abandonado y frustrado. La forma globosa pura infantil del follaje destaca el signo de retardo que al dibujar ramos con redondeles de un tercio de la altura del tronco, demuestran la sentimentalidad, pero también al estar cerrado y vacío una agresividad que no se manifiesta. La tendencia un tanto obsesiva a la repetición se destaca de los detalles monótonos. Los accesorios en concreto el dibujado en la parte izquierda del árbol como cayéndose, reiteran la sensibilidad o la renuncia a algo, abandonar algo, tal vez la pérdida de la personalidad, y tener que soportar un sufrimiento, si bien los frutos estereotipados expresan la falta de capacidad y de independencia en el juicio.

Conflictos: Las líneas rectas y curvas preferentemente manifiestan la introversión, pero también los suaves contactos, que al estar dibujado el árbol en líneas sueltas, estas expresan la reserva y desconfianza, así como la búsqueda de novedades. La porosidad del trazo denota la transigencia y permeabilidad del carácter. La posición izquierda total en la parte mitad superior nos dice que la sujeción ambivalente al pasado, a la madre y a cuanto representa su imagen, pero también la falta de confianza. El tamaño es pequeño que demuestra el sentimiento de inferioridad, y las alturas y anchura comprenden la puerilidad con sueños de potencia compensadora, así como la falta de reflexión y control permaneciendo a la defensiva de manera crispada. Los redondeles ennegrecidos demuestran la insatisfacción de las necesidades afectivas, y los espacios vacíos remarcan dicho sentido de inferioridad que por el ennegrecimiento de aquellos detalles revelan los sueños repetitivos que facilitan la compensación pero no anulan la tristeza. La forma impropia e irregular así como la deformidad del árbol dibujado, muestran que el dibujante no ha encontrado aún su forma propia, y si la tuvo trata de disimularla con un "disfraz", también esta impropiedad muestra el estado de turbación, oculta algo, así como la desmesura y falta de control en las manifestaciones y en las apreciaciones. Su estilo es incompleto, torpe y deforme, sugiriendo la falta de racionalización de los impulsos, luchas afectivas y el control de las mismas, con obturación afectiva y desequilibrio de la personalidad. 
V. Interpretación diagnóstica: Deterioro leve sobre un nivel de inteligencia muy limitado. Labilidad afectiva en relación con el deterioro.

\section{DISCUSIÓN:}

En relación a Olga G.C. nos parece de interés lo siguiente. Por su edad no le hemos aplicado el I6-PF de Catell. Por ello, para el conocimiento de su manera de ser, situación personal y familiar en este momento nos hemos valido, además de los datos extraídos de la entrevista personal, del análisis formal y dinámico de los dibujos que nos realizó. En el presente artículo comentamos específicamente los dos árboles dibujados por Olga, que aparecen numerados como $n^{\circ} \mid y^{\circ} 2$.

Como se ha dicho anteriormente el dibujo $n^{\circ}$ I, fue lo primero que solicitamos a Olga tras entrar en el despacho en el que se celebró la entrevista. La niña se mostraba hostil, nos mira con desconfianza y se manifiesta en actitud defensiva y poco comunicativa. Cuando le decimos: "Me han dicho que dibujas muy bien ¿Quieres dibujarme un árbol frutal?'. cogió el lápiz y el folio y de forma inmediata, con trazos seguros y sin titubeos realizó el dibujo que comentamos. Al estar sentada frente a la perito, el árbol dirige la parte superior de su copa directamente hacia esta persona, y por tanto el penacho puntiagudo con que la termina. Por ello, en nuestra opinión, esa terminación punzante tiene que ser interpretada aquí como clara manifestación de agresividad inicial hacia la exploración y hacia la persona que la explora e intenta entrometerse en su intimidad.

Cuando continuamos hablando sobre sus actividades en el centro, sus aficiones, etc. Olga va bajando sus resistencias y la comunicación se va haciendo más fluida, es entonces (casi una hora después) cuando le decimos "Ahora me vas a dibujar lo que tu quieras" y a este momento corresponde el segundo árbol que aparece en un dibujo, que engloba la casa y un espacio superior nuboso y con un sol luminoso y sonriente (árbol analizado como $n^{\circ} 2$ ), que en lo esencial es similar al primero (forma del tronco, ramas, inclinación, etc.) pero en el que ha desaparecido el signo agresivo del penacho puntiagudo, que debe interpretarse como una manifestación de agresividad inicial y pasajera.

Como resultante del análisis de los árboles, se puede decir que no padece retraso mental, sino que el ambiente en el que se ha desarrollado no ha favorecido ni estimulado su desarrollo intelectual, ni los intereses por el entorno. Su bajo rendimiento escolar, en este momento, también está en relación con la desintegración global por la que pasa en estos momentos.

Un rasgo común a todos sus dibujos es la regresión. En su evolución se ha fijado a estadios infantiles, no está madurando adecuadamente (árbol dirigido hacia la izquierda o próximo al margen izquierdo), continúa vinculada a su infancia, su madre y su padre (el sol, arriba, pero a la izquierda).

En el dibujo del árbol-casa $\left(n^{\circ} 2\right)$, se muestra claramente el rechazo que hace a enfrentarse al futuro y a la realidad, en el margen derecho aparece un espacio blanco y la casa presenta una línea vertical a modo de muro que se cierra frente al borde del papel que significa el futuro.

La casa aparece con dos puertas pequeñas, sin camino de acceso y tanto estas como las ventanas aparecen cerradas, esto es indicativo de introversión, defensa a que se entre en su intimidad, egocentrismo. El tejado grande y la casa mirando hacia la izquierda significa seguridad en su ambiente familiar y su familia, pero vinculados al pasado ya que se sitúan o miran al margen izquierdo (el árbol y la casa son antropomorfos y se representan mirándose mutuamente). El humo de la chimenea significa este "calor de hogar" dirigido hacia la derecha, como idea de futuro, pero lo representa con clara simbología fálica, tema que para ella es motivo de conflicto. 
El espacio superior, merece un comentario especial. Este es el espacio de las fantasías y en él están representadas unas nubes que síllegan hasta el borde derecho, lo cual interpretamos como una esperanza para ella de llegar a algún sitio (en la entrevista manifestó su deseo de ser maestra).

Como resumen, podemos decir que los dos dibujos que ha realizado de sendos árboles, nos muestran:

$1^{\circ}$. La actitud de agresividad que mostraba inicialmente hacia la persona que iba a explorarla y la situación de la exploración.

$2^{\circ}$. La desaparición de esa agresividad tras una comunicación hábil llevada hacia sus juegos, sus aficiones, etc. retrasando el entrar en la materia de la pericia hasta habernos ganado su confianza.

$3^{\circ}$. Evidencian su sentimiento de soledad, su regresión, temor al futuro y a la realidad, su dificultad para establecer relaciones interpersonales adecuadas, su egocentrismo, su retraso intelectual y afectivo, y todo lo que se ha reseñado en la "Ficha de recogida de datos".

No obstante, consideramos que ella alberga expectativas en el futuro, lo cual sería como una esperanza para su reeducación afectiva y relacional. No será un proceso fácil, porque está muy encerrada en sí misma y en un estadio regresivo, pero con la psicoterapia adecuada el resultado podría ser muy positivo.

Con respecto a Manuel S.M. podemos decir lo siguiente. Como resumen de su perfil de personalidad, evaluada por el I6-PF de Catell, se manifiesta como un hombre frío y poco afectivo. Retraído y al margen de la sociedad. Muy lábil emocionalmente, circunstancia en la que, sin duda influye el deterioro que el alcoholismo le haya podido producir. Dominante e impulsivo, suspicaz, con tendecia a la mentira. En conflicto consigo mismo, aunque con un bajo nivel de ansiedad (seguramente esta está mitigada por su consumo de alcohol y su baja autocrítica).

El análisis del Test del Árbol dibujado por Manuel mostraba entre otras características personales las siguientes: estado regresivo, debilidad mental o deterioro, aislamiento, egocentrismo, tendencia a la mentira, ocultación y cobardía. Introversión, inhibición. Dificultad para contactar con la realidad, conflicto social, falta de madurez intelectual y afectiva, agresividad, falta de perseverancia. Deseo de aparentar seguridad en sí mismo, búsqueda de apoyo firme y deseos insatisfechos que el sujeto niega. Carácter introvertido, desconfiado, dureza y frialdad del carácter. Inhibición de impulsos, carencia de sentido vital. Como vemos el Test del Árbol coincide plenamente en los rasgos puestos de manifiesto por el Cuestionario I6-PF, pero va incluso más allá completando algunos de ellos con información muy valiosa en relación al caso que nos ocupa.

Esto ha venido a ratificar el interés de esta prueba en la pericia forense, una vez que el perito se familiarice con la forma de recoger e interpretar los datos, lo cual no encierra mucha dificultad si se siguen las directrices del libro propuesto.

Los resultados obtenidos, no tienen en este caso un especial interés terapeútico, dadas las circunstancias personales de Manuel S.M., y sobre todo su edad, su alcoholismo, su situación de aislamiento (medio rural, trabajo en el campo en solitario, etc.). No consideramos que sea peligroso como agresor sexual, pero su deterioro moral y social sí lo han llevado probablemente a aprovecharse de la situación circunstancial de contacto con la niña y que se ha recogido en el presente artículo.

En cuanto a Jesús D.O., de 78 años, el Cuestionario de Personalidad I6-PF de Catell había puesto de manifiesto que es un hombre con un nivel de inteligencia bajo y cuyos intereses se limitan al cuidado de algunos animales. Es tímido y reservado, inestable emocionalmente y muy sensi- 
ble y afectable por los sentimientos. Es posible que su inestabilidad emocional y sensibilidad hayan aumentando en los últimos años, debido a la edad, ya que la vejez produce a menudo una mayor sensibilidad, y labilidad-inestabilidad afectiva. Su nivel de ansiedad-excitación es bajo, aunque su aislamiento social es importante y se ha acentuado con los acontecimientos, ya que siente vergüenza de conectar con el vecindario.

El Test del Árbol realizado por Jesús ha mostrado, como consta más ampliamente en la ficha, debilidad mental, probablemente acentuada por cierto grado de deterioro senil, comportamiento y curiosidad infantil. Impulsividad y emotividad que le originan un conflicto social. Gran inseguridad interior, aislamiento del mundo circundante. Reserva, desconfianza. Sujeción al pasado. Dificil control de la fantasía. Carácter pueril, miedo a la vida real. Sentimientos de inferioridad. Está confundido y huye de la realidad, se siente frustrado y abandonado. Falta de capacidad de independencia y juicio. Falta de control, cerrado en sí mismo y crispado.

Como en el caso anterior es un resultado coherente con el Cuestionario I6-PF y, al mismo tiempo amplía la información sobre el estado en que se encuentra la persona en el momento actual.

Todo ello nos lleva a insistir como conclusión en la utilidad del Test del Árbol, como prueba de exploración psicodiagnóstica, por su economía de tiempo y de medios. No se puede olvidar que el solicitar un dibujo es una buena forma de iniciar un contacto que, a menudo está sujeto a suspicacia, resistencia y obstrucción. Sin embargo, la persona dificilmente puede adivinar que el simple dibujo de un árbol pueda a posteriori proporcionar tanta información sobre ella misma. La faceta de servir como medio para facilitar la comunicación se ve complementada por la amplitud e importancia de datos sobre la estructura de personalidad, actitudes, nivel de inteligencia, conflictividad social y personal, impulsividad y agresividad, así como el control de la misma, etc, que el análisis proporciona. La experiencia continuada con el Test siguiendo fielmente las instrucciones de interpretación propuestas en el presente artículo irán proporcionando al perito un progresivo rendimiento en el análisis de esta prueba.

\section{BIBLIOGRAFIA:}

I. CASTELLANO ARROYO, Ma. Interés médico-legal de los malos tratos domésticos. Communio. Vol.XXXIV//julio-diciembre 2001.

2. KOCH, K. Der Baumtest. Hans Huber. Berna, 1957. Citado por M. LUND EDELWEISS. En Los Tests : Manual de Técnicas de exploración psicológica. Béla Székely. 5 a ed. Editorial Kapeluz. Buenos Aires 1966. 3. KOCH, K. El Test del Arbol. Editorial Kapeluz. Buenos Aires, 1962. 4. STORA, R. El Test del Árbol. Ed. Paidos. Barcelona, 1989. 5. PASSI, D. Metodi e tecniche nella diagnosi della personalitá. Giunti Barbéra. Firenze 1978.

6. ABAD ALEGRía, F. Empleo Práctico del Test del Árbol en niños y adolescentes (Un renovado método diagnóstico). Secretariado de Publicaciones de la Universidad de Zaragoza, 1985.
7. XANDRÓ, M. Manual de Test Gráficos. Editorial EOS. Madrid, 1987.

8. CID RODRIGUEZ, J.Ma . El Tests del Árbol: Evaluación Psicológica (Índice de variables e interpretación). Granada 1998. 9. CID RODRIGUEZ, J.M y y CASTELLANO ARROYO. El Tests del Árbol en mujeres víctimas de violencia familiar. Cominicación a las VIII ${ }^{\mathrm{a}}$ Jornadas de psicoanálisis en la Universidad. Lleida, marzo-abril de 2000.

10. CATELL, R.B. 16-PF. Cuestionario Factorial de Personalidad (adolescentes y adultos). I5 edición. TEA Ediciones S.A. Madrid 1998. 